\title{
Impact of regional pre-hospital emergency medical services in treatment of patients with acute ischemic stroke
}

\author{
Cemal B. Sozener and William G. Barsan \\ University of Michigan Medical School, Department of Emergency Medicine, Ann Arbor, Michigan
}

Address for correspondence: Cemal B. Sozener, University of Michigan Medical Center, Department of Emergency Medicine, Taubman Center B1 354, 1500 E. Medical Center Dr., SPC 5303, Ann Arbor, MI 48109. cemal@med.umich.edu

Stroke is a major public health concern afflicting an estimated 795,000 Americans annually. The associated morbidity and mortality is staggering. Early treatment with thrombolytics is beneficial. The window for treatment is narrow and minimization of the time from symptom onset to treatment is vital. The general population is not well informed as to the warning signs or symptoms of stroke, leading to substantial delays in emergency medical services (EMS) activation. Ambulance transport of stroke patients to the hospital has demonstrated improvements in key benchmarks such as door to physician evaluation, door to CT initiation, and increased thrombolytic treatment. Pre-hospital notification of the impending arrival of a stroke patient allows for vital preparation in the treating emergency department, and improving timely evaluation and treatment upon arrival of the stroke patient. EMS systems are a vital component of the management of stroke patients, and resources used to improve these systems are beneficial.

Keywords: stroke; emergency medical services (EMS); pre-hospital; t-PA; thrombolytics

\section{Background}

Stroke is a substantial public health challenge. An estimated 795,000 people experience stroke annually, of which $87 \%$ are ischemic. ${ }^{1}$ In 2006 , an estimated 6.4 million Americans 20 years old or older had experienced a stroke within their lifetime. ${ }^{1}$ The mortality associated with stroke is devastating, accounting for one in every 18 deaths, making it the fourth leading killer of Americans and the second leading cause of death worldwide. ${ }^{2}$ Of equal concern is that stroke is the leading cause of serious, long-term adult disability in the United States. ${ }^{3}$ Fifteen to $30 \%$ of stroke survivors are left permanently disabled, while $20 \%$ require nursing home care at three months following their stroke. ${ }^{1}$ This leads to direct and indirect healthcare costs estimated at $\$ 73.7$ billion in $2010 .^{1}$ There is an additional loss of worker productivity and wages, further increasing the cost attributed to stroke. The majority of costs are due to rehabilitation and nursing home expenditures. ${ }^{4}$

\section{Stroke treatment}

Currently there is only one approved drug for the treatment of acute ischemic stroke (AIS) intravenous thrombolytic therapy with recombinant tissue-type plasminogen activator (rt-PA). In 1996, the U.S. Food and Drug Administration (FDA) approved the use of rt-PA for the treatment of ischemic stroke in patients presenting within 3 hours of symptom onset. This followed publication of the landmark National Institute of Neurological Disorders and Stroke (NINDS) rt-PA stroke study in which 624 patients with AIS were randomized to placebo or rt-PA $(0.9 \mathrm{mg} / \mathrm{kg}$ dose $)$ within three hours of symptom onset. A $12 \%$ absolute improvement in favorable outcome with rt-PA treatment, compared with placebo, was demonstrated $(20-38 \%) .{ }^{5}$ A similar benefit was realized one year after stroke as well. ${ }^{6}$ Patients treated with rt-PA were at increased risk of developing intracranial hemorrhage (6.4\%) compared with placebo $(0.6 \%)$; however, the overall mortality was similar despite 
this risk at three-month and one-year follow-ups. ${ }^{5,6}$ In 2008, the European Cooperative Acute Stroke Study (ECASS)-3 trial demonstrated improved clinical outcomes in select patients who were treated with rt-PA in the 3- to 4.5-h window; however, this benefit was not as substantial compared with those treated earlier. ${ }^{7,8}$ While not yet FDA approved for this expanded time horizon, rt-PA treatment is recommended in selected patients up to $4.5 \mathrm{~h}$ following symptom onset by the American Stroke Association (ASA). ${ }^{8}$

For acute stroke patients, it is very clear that "time lost is brain lost." Thrombolytic therapy can significantly improve patient outcome when given as soon as possible after onset of stroke symptoms. ${ }^{9}$ NINDS recommendations published in 1996 outline time goals for patients being evaluated in the emergency department (ED) for acute ischemic stroke. These benchmarks are $10 \mathrm{~min}$ for door to ED physician evaluation, $15 \mathrm{~min}$ for door to stroke team or neurologist notification, $25 \mathrm{~min}$ for door to computed tomography (CT) scan initiation, $45 \mathrm{~min}$ for door to expert interpretation of the CT scan, and $60 \mathrm{~min}$ for door to thrombolytic administration. ${ }^{10}$ Aggressive early management in the emergency department can limit the morbidity associated with this deadly disease process. Despite the clear benefits of treatment with rt-PA, currently only $2-5 \%$ of patients with ischemic stroke receive thrombolytic therapy. ${ }^{11}$ Delayed recognition of stroke symptoms and delayed arrival to the hospital are major reasons for exclusion from treatment. ${ }^{11,12}$ Basing our conclusions on research performed by the California Acute Stroke Pilot Registry Investigators, if patients with a known time of symptom onset had immediately called 911, we submit that expected treatment with thrombolytics within three hours of symptom onset would have increased from 4.3 to $28.6 \%{ }^{13}$ The mode of transportation to the hospital is strongly associated with in-hospital delays in evaluation. Studies have demonstrated that patients with presumed stroke arriving to the hospital via EMS were more likely to have brain imaging performed and interpreted in a timely fashion than patients arriving by private transportation. ${ }^{11}$ Therefore, improving the patient's understanding of symptoms of stroke with rapid activation of the EMS system and subsequent rapid transport of the patient to the hospital can have a substantial impact on rates of treatment in acute stroke.

\section{Patient activation of EMS}

Early patient recognition of signs or symptoms of stroke and transport via EMS would have the greatest impact on increasing thrombolytic therapy in the appropriate patient population. ${ }^{13}$ In 2005 , surveys of patients in 14 states revealed that only $38 \%$ were aware of five warning symptoms of stroke and would call 911 if they thought someone was having a stroke. Awareness of these warning signs and the plan to call 911 were greater in whites versus blacks and Hispanics, women versus men, and those with college degrees versus those without a high school diploma. ${ }^{14}$ In a study of 163 patients admitted to an emergency room (ER) with possible stroke, 39\% did not know a single sign or symptom. ${ }^{1}$ It has been demonstrated that symptom severity is the single most important patient-dependent determinant of net pre-hospital delay. ${ }^{12}$ Severe stroke presentations are associated with earlier EMS activation. Because of the time required in the ER to completely evaluate and assess patients experiencing a stroke before being able to appropriately treat them with t-PA (approximately one hour), early EMS activation is paramount. In addition to potentially increasing treatment rates, presenting to the hospital as soon as possible following onset of stroke symptoms would lead to earlier administration of t-PA, which has been shown to be more effective. ${ }^{9}$ Improving public education regarding stroke symptoms and the narrow therapeutic time horizon could have a profound effect on increasing thrombolytic treatment rates; ${ }^{15,16}$ however, this type of widespread intervention and public health campaign would likely be very costly. Because of patients' lack of understanding of stroke signs and symptoms, the burden of having a high index of suspicion for stroke is transferred to EMS personnel and dispatchers. ${ }^{17}$

\section{EMS dispatch}

Timely and appropriate care from EMS can play a major role in reducing the time from stroke onset to thrombolytic treatment. Data indicate that between 29 and $65 \%$ of patients with signs or symptoms of stroke contact the health system via EMS. ${ }^{11,18}$ EMS dispatchers are the first link in the chain leading to hospital presentation for many stroke patients. It is imperative that dispatchers are well trained to detect stroke symptoms from the initial call in addition to appropriate triage and prioritized dispatch of emergency medical personnel to the location. ${ }^{18}$ Training 
programs for EMS dispatchers have shown that dispatch accuracy for stroke can be improved. ${ }^{19,20}$

\section{EMS assessment}

The time-sensitive nature of the treatment of acute ischemic stroke has important implications for paramedics. They must be proficient in their ability to recognize, assess, manage, treat, and transport stroke patients. ${ }^{17}$ Once the ambulance arrives on the scene, the EMS provider should obtain key historical details. Accurate identification of the time of symptom onset is paramount. EMS providers may have the best chance of obtaining the most accurate history because of access to family, caregivers, or bystanders who may not be immediately available once the patient arrives in the ED. ${ }^{17}$ After initial patient stabilization, a focused examination should evaluate for common presenting signs of stroke. ${ }^{18}$ Prehospital stroke assessment tools such as the Cincinnati Pre-hospital Stroke Scale and the Los Angeles Pre-hospital Stroke Screen effectively identify stroke patients in the field. ${ }^{21,22}$ These instruments have demonstrated sensitivities of greater than $90 \%{ }^{21,22}$ and should be used for the assessment of all patients considered to be having a stroke. ${ }^{23}$

\section{EMS management}

Once initial stabilization has occurred, the American Stroke Association (ASA) guidelines for EMS management of stroke patients state that transport to the closest institution that provides emergency stroke care should occur without delay. ${ }^{18}$ En route, emergency medical personnel should manage airways, breathing and circulation, initiate cardiac monitoring and electrocardiogram (EKG), establish intravenous (i.v.) access, provide oxygen to maintain a saturation greater than $92 \%$, perform a glucose measurement, and keep the patient NPO (nothing by mouth). ${ }^{18}$ The avoidance of supplemental oxygen in nonhypoxic patients has been suggested by previous clinical trials of hospital patients, although the use of supplemental oxygen for pre-hospital stroke patients has not been evaluated in large-scale clinical trials. ${ }^{24,25}$ The development of stroke protocols for EMS personnel is strongly encouraged to consistently and aggressively treat these patients.

\section{Hospital notification}

Advanced notification to the treating facility of an incoming stroke patient by EMS has been shown to be very beneficial in providing necessary prepara- tion time to hospital personnel. ${ }^{26}$ Early notification can be used to optimize downstream processes by alerting stroke teams, reserving CT scanners, notifying radiology personnel of impending stat head CT imaging requiring interpretation, making pharmacists aware of the potential need for thrombolytics, and improving chances for rapid evaluation of the patient even during times of high ED volume and occupancy. ${ }^{18,27}$ Pre-hospital notification by EMS as compared to no notification was positively associated with neuroimaging completed within 25 min of arrival and interpretation within $45 \mathrm{~min}$ of arrival, which are in line with American Heart Association (AHA) guidelines. ${ }^{11}$ This early notification has been found to increase the percentage of patients with acute stroke who receive thrombolytic therapy. ${ }^{11,28}$ Pre-hospital notification also leads to decreased delays from ED arrival to neurological assessment and initial brain imaging in patients brought in by EMS. ${ }^{17}$ Communication between paramedics and hospitals is paramount, and while it is routine for paramedics to be in contact with receiving facilities while en route, regardless of the patient condition, it is crucial that hospitals have systems in place to maximize the use of this information when it is received, similar to successes observed with the pre-hospital activation of cardiac catheterization labs. A movement from pre-hospital notification of the arrival of stroke patients to pre-hospital activation of stroke teams is likely to be of the highest yield.

\section{Destination hospital}

It is recommended that EMS providers rapidly deliver stroke patients to primary stroke centers (PSCs). Several studies have documented improved patient outcomes when stroke patients are admitted to a stroke unit or receive specialized stroke care. ${ }^{23}$ Regional policies facilitating the transport of patients to a PSC have demonstrated increased thrombolytic use in appropriate patient populations. ${ }^{29-31}$ Regional stroke systems should be used that implement policies and regulations to ensure that stroke patients are transported to a PSC regardless of the patient's location. ${ }^{32}$ The patient should be transported to the nearest PSC if it is located within a reasonable transport distance and transport time. ${ }^{32}$

\section{Air medical transport}

In some instances, stroke patients are in remote geographical locations with no local hospital facilities 
making it difficult for ground-based EMS systems to deliver them to stroke centers in a timely fashion. In these cases, use of air medical transport should be considered. ${ }^{33}$ Given the current FDA-approved 3-h time window for thrombolytic therapy, if ground transportation is likely to take more than an hour, it will likely preclude the ability to administer i.v. rt-PA. ${ }^{17}$ In these cases, protocols should be in place to transport these patients to stroke centers by air ambulance if this will allow treatment within the time horizon. The use of air medical transport could potentially reduce transport time, increase availability of thrombolytic therapy to those living in rural communities, and be cost effective. ${ }^{33-36}$

\section{Pre-hospital delivery of stroke treatment}

With advancing science, there is interest in the development of neuroprotective therapies that may be used in the early treatment of acute ischemic stroke. Much of the research using these agents has been hindered by delayed time-to-delivery after the onset of stroke symptoms. There is a natural interest in researching whether these agents can be successfully deployed in the pre-hospital phase. This may be beneficial when transport times are long due to lack of nearby medical centers and particularly in rural regions. If patients could be accurately identified as having a stroke by EMS personnel, paramedics could be trained to deliver these agents in the back of an ambulance during transport. This would be far more efficient than waiting until the patient reaches the medical center and is evaluated. In the Field Administration of Stroke Therapy-Magnesium (FASTMAG) pilot trial, the research group studied the safety and feasibility of paramedic initiation of intravenous magnesium sulfate in the field. ${ }^{37}$ They determined that using tools to rate neurologic deficits, such as the National Institutes of Health Stroke Scale (NIHSS), was overly time-consuming and unwieldy to deploy in the pre-hospital setting. ${ }^{37}$ Instead, they used the Los Angeles Motor Scale (LAMS), which rates severity of face, arm, and leg weakness on a zero- to five-point scale. ${ }^{38}$ It is derived directly from the Los Angeles Pre-hospital Stroke Screen (LAPSS) and does not require additional evaluation time to use, as the paramedics would have already administered this tool at the start of their patient evaluation. The LAMS was found to have excellent concurrent validity compared with the NIHSS, excellent interrater reliability, and good long-tem outcome predictive validity. ${ }^{37}$ The results of this study demonstrated that enrollment in the trial and implementation procedures did not increase the EMS scene to ED transport times. They further found that the initiation of magnesium sulfate in the field is safe, feasible, and permits hyperacute delivery of neuroprotective therapy. ${ }^{37}$ A phase III trial of prehospital magnesium for stroke patients with definite motor deficits is ongoing in southern California.

\section{Access to stroke care}

Stroke care in the United States can be highly variable based on geographical location. In 2002, approximately $21 \%$ of U.S. counties did not have a hospital, 31\% lacked a hospital with an ED, and $77 \%$ had a hospital lacking neurological services. ${ }^{1} \mathrm{~A}$ recently published study found that $62 \%$ of U.S. hospitals did not report treating any patients with t-PA during the two-year study period. ${ }^{39}$ This lack of nearby emergency neurological services in times of acute stroke showcases the need for well-established stroke systems of care that are often regional or even state wide. EMS response and transportation protocols must be established and in place for survival of these patients in critical neurologic situations. These systems often mirror those in place for other time-sensitive disease processes such as trauma and acute myocardial infarction.

\section{Stroke systems}

As stroke is a time-sensitive disease process and the window for intervention is very restrictive, the development of stroke systems of care is paramount. These concepts engulf more than single hospitals and should be developed as regional or statewide systems. The availability of resources to care for acute stroke patients varies widely both among and within communities. ${ }^{18}$ The resources, personnel involved, infrastructure, and training required for optimizing these systems are substantial and require advance planning, and frequent updating. EMS medical directors, community leaders, physicians, and hospital administrators should be active participants, if not leaders, in the development of these stroke systems. ${ }^{18,23}$ These stroke systems must address issues related to communication, transportation, access to care, transfer of patients, sharing of resources across EMS systems, and provide systematic review and evaluation. ${ }^{32}$ To be successful, these stroke systems should use protocols, tools, and 
training that meet current AHA/ASA guidelines for stroke care. ${ }^{32}$ Seamless teamwork between the EMS and ED staff is one of the key issues in forming a successful chain of recovery for acute stroke. ${ }^{12}$

\section{Conclusion}

Stroke is a substantial public health concern with profound mortality and devastating morbidity. Thrombolytic therapy is the sole approved treatment for acute ischemic stroke; however, treatment must be initiated within a narrow window. In 2009, only $3.4-5.2 \%$ of patients with acute ischemic stroke were treated with rt-PA. ${ }^{40}$ Delayed presentation to the hospital is the primary reason patients are not receiving thrombolytic treatment. Once patients arrive in the $\mathrm{ER}$, the testing and interpretation required to safely and appropriately administer t-PA takes valuable time in an already very narrow window. Activation of an EMS system optimized to promptly and accurately evaluate stroke patients and deliver them to the nearest stroke center with early notification to the accepting facility will improve outcomes in stroke. Unfortunately, the general public has limited understanding of signs and symptoms associated with stroke, placing the burden of suspecting stroke on EMS personnel. In order for pre-hospital care to be successful, there needs to be a well-established regional system for stroke care with thorough training to ensure AHA/ASA guidelines for stroke care are met. There may also be a future role for paramedics to provide neuroprotective agents to patients during transport to the stroke center. EMS personnel have an important role in the management of the acute stroke patient. Decisions made by dispatchers and paramedics can affect the treatment and contribute to the outcome of stroke patients. Seamless cooperation between pre-hospital providers and ED staff will lead to improvements in the care of stroke patients.

\section{Conflicts of interest}

The authors declare no conflicts of interest.

\section{References}

1. Lloyd-Jones, D. et al. 2010. Heart disease and stroke statistics-2010 update: a report from the American Heart Association. Circulation 121: e46-e215.

2. Towfighi, A. \& J.L. Saver. 2011. Stroke declines from third to fourth leading cause of death in the United States: historical perspective and challenges ahead. Stroke 42: 2351-2355.

3. McNeil, J.M. 2001. Prevalence of disabilities and associ- ated health conditions among adults-United States, 1999. MMWR Morb. Mortal Wkly Rep. 50: 120-125.

4. Fagan, S.C. et al. 1998. Cost-effectiveness of tissue plasminogen activator for acute ischemic stroke. NINDS rt-PA Stroke Study Group. Neurology 50: 883-890.

5. The National Institute of Neurological Disorders and Stroke rt-PA Stroke Study Group. 1995. Tissue plasminogen activator for acute ischemic stroke. N. Engl. J. Med. 333: 15811587.

6. Kwiatkowski, T.G. et al. 1999. Effects of tissue plasminogen activator for acute ischemic stroke at one year. National Institute of Neurological Disorders and Stroke Recombinant Tissue Plasminogen Activator Stroke Study Group. N. Engl. J. Med. 340: 1781-1787.

7. Hacke, W. et al. 2008. Thrombolysis with alteplase 3 to 4.5 hours after acute ischemic stroke. N. Engl. J. Med. 359: 13171329.

8. Del Zoppo, G.J. et al. 2009. Expansion of the time window for treatment of acute ischemic stroke with intravenous tissue plasminogen activator: a science advisory from the American Heart Association/American Stroke Association. Stroke 40: 2945-2948.

9. Lees, K.R. et al. 2010. Time to treatment with intravenous alteplase and outcome in stroke: an updated pooled analysis of ECASS, ATLANTIS, NINDS, and EPITHET trials. Lancet 375: 1695-1703.

10. Evenson, K.R. et al. 2009. A comprehensive review of prehospital and in-hospital delay times in acute stroke care. Int. J. Stroke 4: 187-199.

11. Patel, M.D. et al.2011. Prehospital notification by emergency medical services reduces delays in stroke evaluation: findings from the North Carolina stroke care collaborative. Stroke 42: 2263-2268.

12. Puolakka, T. et al. 2010. Sequential analysis of pretreatment delays in stroke thrombolysis. Acad. Emerg. Med. 17: 965969.

13. Johnston, S.C., K. Fong \& N. Hills. 2005. Prioritizing interventions to improve rates of thrombolysis for ischemic stroke. Neurology 64: 654-659.

14. Fang, J., N.L. Keenan \& C. Ayala. 2008. Awareness of stroke warning symptoms-13 States and the District of Columbia, 2005. MMWR Morb. Mortal. Wkly Rep. 57: 481485.

15. Williams, L.S. et al. 1997. Stroke patients' knowledge of stroke. Influence on time to presentation. Stroke 28: 912915.

16. Lacy, C.R. et al. 2001. Delay in presentation and evaluation for acute stroke: Stroke Time Registry for Outcomes Knowledge and Epidemiology (S.T.R.O.K.E.). Stroke 32: 63-69.

17. Crocco, T.J. et al. 2007. EMS management of acute strokeprehospital triage (resource document to NAEMSP position statement). Prehosp. Emerg. Care 11: 313-317.

18. Adams, H.P., Jr. et al. 2007. Guidelines for the early management of adults with ischemic stroke: a guideline from the American Heart Association/American Stroke Association Stroke Council, Clinical Cardiology Council, Cardiovascular Radiology and Intervention Council, and the Atherosclerotic Peripheral Vascular Disease and Quality of Care Outcomes in Research Interdisciplinary Working Groups: The American Academy of Neurology affirms the value of this 
guideline as an educational tool for neurologists. Circulation 115: e478-534.

19. Kothari, R. et al. 1995. Frequency and accuracy of prehospital diagnosis of acute stroke. Stroke 26: 937-941.

20. Handschu, R. et al. 2003. Emergency calls in acute stroke. Stroke 34: 1005-1009.

21. Kothari, R.U. et al. 1999. Cincinnati Prehospital Stroke Scale: reproducibility and validity. Ann. Emerg. Med. 33: 373378.

22. Kidwell, C.S. et al. 2000. Identifying stroke in the field. Prospective validation of the Los Angeles prehospital stroke screen (LAPSS). Stroke 31: 71-76.

23. Millin, M.G., T. Gullett \& M.R. Daya. 2007. EMS management of acute stroke-out-of-hospital treatment and stroke system development (resource document to NAEMSP position statement). Prehosp. Emerg. Care 11: 318-325.

24. Ronning, O.M. \& B. Guldvog. 1999. Should stroke victims routinely receive supplemental oxygen? A quasi-randomized controlled trial. Stroke 30: 2033-2037.

25. Singhal, A.B. et al. 2005. A pilot study of normobaric oxygen therapy in acute ischemic stroke. Stroke 36: 797-802.

26. National Institute of Neurological Disorders and Stroke (U.S.) Symposium (2nd: 2002: Arlington, VA) \& National Institute of Neurological Disorders and Stroke (U.S.). 2003. Improving the Chain of Recovery for Acute Stroke in Your Community: Task Force Reports. National Institutes of Health, Dept. of Health and Human Services. Bethesda, MD.

27. Gomez, C.R. et al. 1994. Code stroke. An attempt to shorten inhospital therapeutic delays. Stroke 25: 1920-1923.

28. Jauch, E.C. et al. 2010. Part 11: adult stroke: 2010 American Heart Association Guidelines for Cardiopulmonary Resuscitation and Emergency Cardiovascular Care. Circulation 122: S818-S828.

29. Morgenstern, L.B. et al. 2002. Improving delivery of acute stroke therapy: the TLL Temple Foundation Stroke Project. Stroke 33: 160-166.

30. Lattimore, S.U. et al. 2003. Impact of establishing a primary stroke center at a community hospital on the use of thrombolytic therapy: the NINDS Suburban Hospital Stroke Center experience. Stroke 34: e55-e57.

31. Alberts, M.J. et al. 2000. Recommendations for the establishment of primary stroke centers. Brain Attack Coalition. JAMA 283: 3102-3109.

32. Acker, J.E., 3rd et al. 2007. Implementation strategies for emergency medical services within stroke systems of care: a policy statement from the American Heart Association/American Stroke Association Expert Panel on Emergency Medical Services Systems and the Stroke Council. Stroke 38: 3097-3115.

33. Schwamm, L.H. et al. 2005. Recommendations for the establishment of stroke systems of care: recommendations from the American Stroke Association's Task Force on the Development of Stroke Systems. Stroke 36: 690-703.

34. Silliman, S.L. et al. 2003. Use of a field-to-stroke center helicopter transport program to extend thrombolytic therapy to rural residents. Stroke 34: 729-733.

35. Chalela, J.A. et al. 1999. Safety of air medical transportation after tissue plasminogen activator administration in acute ischemic stroke. Stroke 30: 2366-2368.

36. Silbergleit, R., P.A. Scott \& M.J. Lowell. 2003. Costeffectiveness of helicopter transport of stroke patients for thrombolysis. Acad. Emerg. Med. 10: 966-972.

37. Saver, J.L. et al. 2004. Prehospital neuroprotective therapy for acute stroke: results of the Field Administration of Stroke Therapy-Magnesium (FAST-MAG) pilot trial. Stroke 35: e106-e108.

38. Llanes, J.N. et al. 2004. The Los Angeles Motor Scale (LAMS): a new measure to characterize stroke severity in the field. Prehosp. Emerg. Care 8: 46-50.

39. Kleindorfer, D. et al. 2009. US geographic distribution of rtPA utilization by hospital for acute ischemic stroke. Stroke 40: 3580-3584.

40. Adeoye, O. et al. 2011. Recombinant tissue-type plasminogen activator use for ischemic stroke in the United States: a doubling of treatment rates over the course of 5 years. Stroke 42: 1952-1955. 